\title{
Visuospatial working memory influences the interaction between space and time
}

\author{
Ariel Starr $^{1}$ - Elizabeth M. Brannon ${ }^{2}$
}

Published online: 26 April 2016

(C) Psychonomic Society, Inc. 2016

\begin{abstract}
How do representations of space inform our perception of time? In language, spatial vocabulary is frequently used to describe temporal concepts, and spatial information biases temporal perception even in non-verbal tasks. In contrast, temporal information typically exerts little, if any, influence on the perception of spatial extent. Here, we used spatial and temporal reproduction tasks, both with and without verbal and spatial dual tasks, to investigate the mechanism underlying the asymmetric relation between space and time. Specifically, we tested whether the asymmetric interference between spatial and temporal stimulus attributes arises from interference in verbal or visuospatial working memory. We found that loading visuospatial working memory, but not verbal working memory, eliminated the asymmetric pattern of interference. This suggests that the interference between spatial and temporal representations arises due to processing constraints in visuospatial working memory. These findings are discussed in terms of the load theory of attention and the relative automaticity with which spatial and temporal information is processed.
\end{abstract}

Keywords Spatial cognition $\cdot$ Metaphor theory $\cdot$ Magnitude processing

Across cultures and languages, spatial vocabulary is commonly co-opted to refer to time (e.g., "she went on a long vacation,

Ariel Starr

arielstarr@berkeley.edu

1 Helen Wills Neuroscience Institute, University of California at Berkeley, Berkeley, CA, USA

2 Department of Psychology, University of Pennsylvania, Philadelphia, PA, USA
" "she tried to put the past behind her"), whereas temporal terms are rarely used to refer to space (Lakoff \& Johnson, 1980). What can the inherent asymmetry in these metaphors reveal about the nature of the underlying correspondence between spatial and temporal representations? According to metaphor theory, concrete domains such as space can be used to ground representations of more abstract domains such as time, therefore aiding in the construction of richer representations of abstract concepts (Lakoff \& Johnson, 1980). Proponents of metaphoric structuring theory suggest that representations of time lack the structure required to support higher-level temporal reasoning. Metaphoric structuring may therefore provide the necessary framework for bolstering sophisticated temporal reasoning (Casasanto \& Boroditsky, 2008; Lakoff \& Johnson, 1980; Srinivasan \& Carey, 2010).

The association between space and time likely extends beyond language to their neural underpinnings (Cantlon, Platt, \& Brannon, 2009; Hubbard, Piazza, Pinel, \& Dehaene, 2005; Walsh, 2003). According to A Theory of Magnitude (ATOM; Walsh, 2003), space and time, as well as number, are jointly represented in the parietal cortex as a result of the role that these dimensions play in sensorimotor transformations necessary for action. Indeed, functional neuroimaging reveals that representations of these dimensions engage overlapping or neighboring regions of posterior parietal cortex (Cohen Kadosh et al., 2005, 2007; V. Dormal, Dormal, Joassin, \& Pesenti, 2011; Fias, Lammertyn, Reynvoet, Dupont, \& Orban, 2003). These findings provide mechanistic support for the many studies that have documented behavioral and neurological links between representations of space, time, and number (Allman, Pelphrey, \& Meck, 2012; Cantlon et al., 2009; Cohen Kadosh, Lammertyn, \& Izard, 2008; Feigenson, 2007; Hubbard et al., 2005; Walsh, 2003). However, implicit in ATOM is the idea that the links between different dimensions should be symmetrical. For example, in the classic size congruity paradigm, 
physical size interferences with judgments of numerical magnitude and numerical magnitude interferences with judgments of physical size (Tzelgov, Meyer, \& Henik, 1992). Interestingly, this symmetry in the associations between different dimensions is not always observed.

As predicted by metaphor theory, the relation between representations of space and time appears to be asymmetric. In a variety of tasks, the pattern of interference found between temporal and spatial representations is unidirectional: spatial information biases judgments regarding time, whereas temporal information has no influence on judgments of space (Bottini \& Casasanto, 2013; Casasanto \& Boroditsky, 2008; Casasanto, Fotakopoulou, \& Boroditsky, 2010; V. Dormal \& Pesenti, 2012; Xuan, Zhang, He, \& Chen, 2007). In one series of experiments, participants were instructed to reproduce either the spatial displacement or temporal duration of visually presented lines and dots (Casasanto $\&$ Boroditsky, 2008). Across the experiments, displacement and duration were manipulated orthogonally to each other, meaning that each dimension could serve as a distractor for the other dimension. Participants' judgments about temporal duration were consistently influenced by irrelevant spatial information, such that physically longer lines were judged to have appeared for longer temporal durations than physically shorter lines. However, spatial displacement judgments were unaffected by irrelevant temporal information. This demonstrates that the asymmetric relation between spatial and temporal representations extends beyond linguistic reasoning and influences the basic perceptions of these dimensions. These findings suggest an inherent asymmetry in the mapping between representations of time and space, such that representations of time may be intrinsically dependent on representations of space.

However, an alternative explanation for these asymmetric effects does not necessitate asymmetry in the underlying mapping between spatial and temporal representations. Dormal and Presenti (2012) proposed that asymmetric interference patterns reflect a gradient in the automaticity with which different dimensions are processed. According to this view, spatial information is processed more automatically than temporal information. Therefore, spatial information is more likely to be processed even when it is task-irrelevant, whereas irrelevant temporal information is less likely processed automatically. As a result, irrelevant spatial information is available to interfere with temporal judgments, whereas because irrelevant temporal information is not processed, it is unavailable to interfere with spatial judgments. A similar explanation has been put forward by Santiago and colleagues (2011), who claim that interference between different dimensions arises in working memory, and it is the relative salience of each dimension that determines the direction of interference.

In the present study, we employed a line reproduction task adapted from Casasanto and Boroditsky (2008) in combination with verbal and spatial dual tasks to shed light on the source of the asymmetric interference between spatial and temporal information. We hypothesized that if the asymmetric interference of spatial information on temporal judgments requires online access to linguistic processing, then loading verbal working memory would minimize the effect (cf. Winawer et al., 2007). Alternatively, if the asymmetry stems from competition between processing both spatial and temporal representations in working memory, then loading visuospatial working memory may decrease the likelihood that irrelevant spatial information is automatically processed and consequently minimize the interference effect.

\section{Materials and methods}

\section{Participants}

Data from 20 subjects were included in the final analyses (mean age $=19$ years, range $=18-21$ years, 12 females). All participants were native English speakers who were not regularly exposed to other languages. Data from an additional five subjects were excluded because of computer error $(n=1)$ or because the slope of their space or time estimates in at least one condition was less than .5 , suggesting that they were not paying attention to the task $(n=4)$. All participants gave informed consent to a protocol approved by the local institutional review board.

\section{Materials}

\section{Growing lines}

The task was modeled after the Growing Lines condition in Casasanto and Boroditsky (2008). Lines of varying physical lengths were presented on a computer monitor for varying temporal durations. Lines grew horizontally across the screen, one pixel at a time, from left to right until they reached their complete length. Five line lengths $(200,350,500,650$, and 800 pixels) and five line durations (1000, 2000, 3000, 4000, and $5000 \mathrm{~ms}$ ) were fully crossed to produce 25 different line types. Each line type was presented twice per block in a randomized order. The starting location of the line was jittered horizontally such that the line started between 200 and 400 pixels to the left of the midline of the screen in order to prevent the frame of the monitor from being used as a reference.

Before each line was shown, the participant was cued to the dimension of interest via the presentation of the word "Space" or "Time," accompanied by a ruler or hourglass icon. The cue remained on the screen for 2 seconds. Immediately after, the line grew across the screen. After the line disappeared, a ruler or hourglass icon appeared on the screen. The icons appeared on 
the left side of the screen, and the vertical position was jittered so that the response was displaced both horizontally and vertically from the stimulus. To reproduce the length of the line, the participant clicked once on the ruler icon and then clicked a second time some horizontal distance to the right of the icon, depending on the physical length of the line. To reproduce the duration of the line, the participant clicked once on the hourglass icon, waited until the judged time had elapsed, and clicked the icon a second time. Thus participants clicked the mouse twice for both temporal and spatial judgments.

\section{Dual tasks}

The dual tasks were delayed match-to-sample tasks with verbal or spatial stimuli. Both tasks were adapted from Winawer et al. (2007). The beginning of the verbal and spatial dual task blocks was marked with the phrase "Remember the digits" or "Remember the pattern." Immediately afterwards, a memory target appeared centrally on the screen for 3 seconds. In the verbal dual task, participants were shown a seven-digit numerical string and instructed to silently rehearse the string while performing the growing lines task. After completing five growing lines trials, two digits strings appeared on the screen: the target string and a distractor string that deviated from the target by a single digit. Participants were instructed to click on the string that matched the target. A new target digit string would then appear on the screen. In the spatial dual task, participants were shown a geometric pattern and instructed to maintain it in memory while performing the line reproduction task. The geometric patterns consisted of a $4 \times 4$ array of squares in which four randomly selected squares were filled. After completing five trials of the growing lines task, the target pattern and a distractor pattern that differed from the target in the location of a single colored square appeared on the screen, and participants were instructed to choose the pattern that matched target. A new target geometric pattern would then appear on the screen. Dual task difficulty was calibrated and equated during pilot testing.

\section{Procedure}

Before the test trials, participants completed two practice trials to familiarize themselves with how to produce spatial and temporal responses. Participants completed three task blocks (standard, verbal dual task, and spatial dual task). The order of the blocks was randomized across participants. All blocks contained 50 growing lines trials. Each dual task block contained ten memory probes.

\section{Results}

For each block (standard, verbal dual task, and spatial dual task), we first performed repeated-measures regression analyses to examine the effects of actual line length on estimated length and actual line duration on estimated duration. These analyses confirmed that participants were performing the tasks as instructed (all $\mathrm{R}^{2} \mathrm{~s}>.99, \beta \mathrm{s}>.7$, and $p s<.001$ ). Next we examined the influence of actual line length on estimated line duration and vice versa. In the standard block, line length had a significant effect on estimated duration $\left(\mathrm{R}^{2}=.93, \beta=1.03, p\right.$ $<.001$ ) (Fig. 1). However, line duration did not exert a significant influence on estimated length $\left(\mathrm{R}^{2}=.54, \beta=.007, p=\right.$ .19).

The outcome of the analyses for the verbal dual task block mirrored those of the standard block. Line length again had a significant effect on estimated duration $\left(\mathrm{R}^{2}=.75, \beta=.77, p=\right.$ $.005)$, whereas line duration did not influence estimated length $\left(\mathrm{R}^{2}=.09, \beta=.005, p=.41\right)$ (Fig. 2). However, a different pattern of results was found for the spatial dual task block, as no cross-dimensional interference was observed. There was no effect of line length on estimated duration $\left(\mathrm{R}^{2}=.34, \beta=\right.$ $.38, p=.18$ ), and no effect of line duration on estimated length $\left(\mathrm{R}^{2}=.30, \beta=.002, p=.67\right)$. Therefore, the asymmetric effect of line length on duration was present in the standard and verbal dual task blocks but eliminated by the spatial dual task.

To determine if the different patterns of results found in the verbal and spatial dual task blocks could be attributed to differences in difficulty of the dual tasks themselves, we compared performance across the tasks. Accuracy was high for both dual tasks (91\% for the verbal task and $93.5 \%$ for the spatial task) and there was no statistical difference in performance between the tasks $(\mathrm{t}(19)=-.98, p=.34)$. It is therefore unlikely that differences in dual task difficulty are driving the different interference patterns observed between the verbal and spatial dual task blocks.

Finally, we performed a two-way repeated measures analysis of variance (ANOVA) to compare the effect of line length on estimated duration versus the effect of line duration on estimated length (as indexed by the slope of the regression lines; see Table 1) across the three different task types. This analysis revealed a significant effect of interference direction $\left(\mathrm{F}(1,19)=119.95, p<.001, \eta^{2}=\right.$ .863 ), which indicates that the length of the line biased participants' duration estimates more than the duration of the line biased their length estimates (length: $\mathrm{M}=.68$, SEM $=.06$; duration: $\mathrm{M}=.01$, SEM $=.001$ ). In addition, there was a significant effect of task type $(\mathrm{F}(2,38)=$ 76.74, $p<.001, \eta_{p}^{2}=.802$ ), indicating that the strength of the interference effect was greater in the standard and verbal dual task conditions compared to the spatial dual task condition. There was also a significant task type by direction interaction $\left(\mathrm{F}(2,38)=79.60, p<.001, \eta_{p}^{2}=\right.$ 
Fig. 1 Mean estimates of line length and duration in the standard task. (A) Effect of actual line length on estimated length. (B) Effect of actual line duration on estimated duration. (C) Effect of actual line length on estimated duration. (D) Effect of actual line duration on estimated length.

Error bars indicate SEM
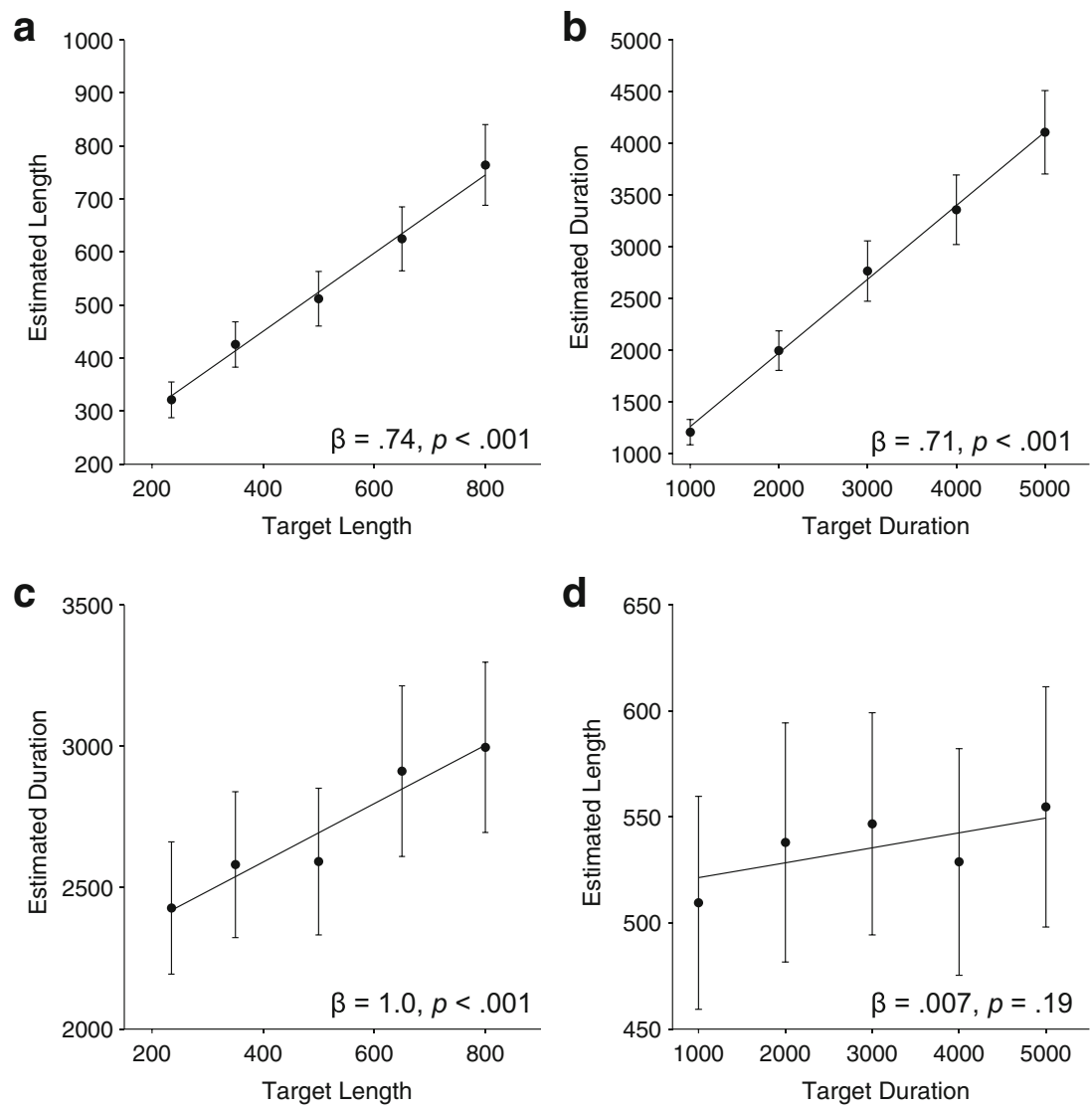

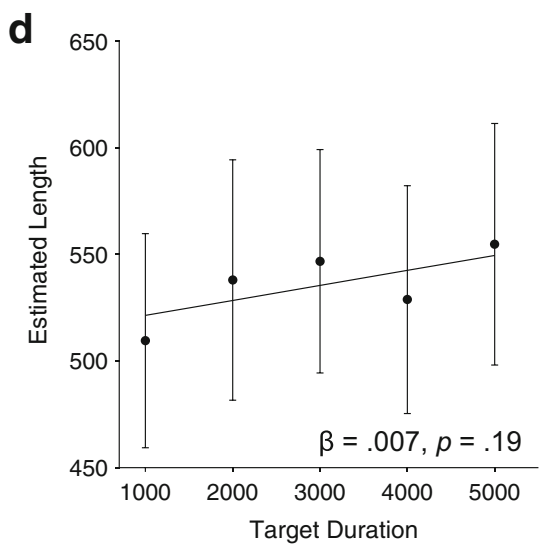

.807), indicating that the difference between the interference in the spatial dual task compared to the verbal dual task and standard task was greater for the effect of line length on estimated duration compared to the effect of duration on estimated line length.

\section{General discussion}

Representations of time are often said to be asymmetrically dependent on representations of space, both in language and in the mind. Many languages employ spatial terms to talk about time, and the nature of these metaphors can influence temporal reasoning (Boroditsky, 2000, 2001; Casasanto, 2008). Furthermore, nonverbal paradigms demonstrate that irrelevant spatial information biases judgments of duration, whereas irrelevant temporal information does not bias judgments of spatial extent (Bottini \& Casasanto, 2010, 2013; Casasanto et al., 2010; Casasanto \& Boroditsky, 2008; V. Dormal \& Pesenti, 2012; Xuan et al., 2007). The current experiment was designed to investigate the source of this asymmetry. Using a reproduction task previously employed by Casasanto and Boroditsky (2008), we replicated the main finding that participants' duration judgments are influenced by irrelevant spatial information, whereas judgments of line length are relatively unaffected by irrelevant temporal
Fig. 2 Effect of actual line length on estimated duration in the verbal dual task (left) and in the spatial dual task (right). Error bars indicate SEM
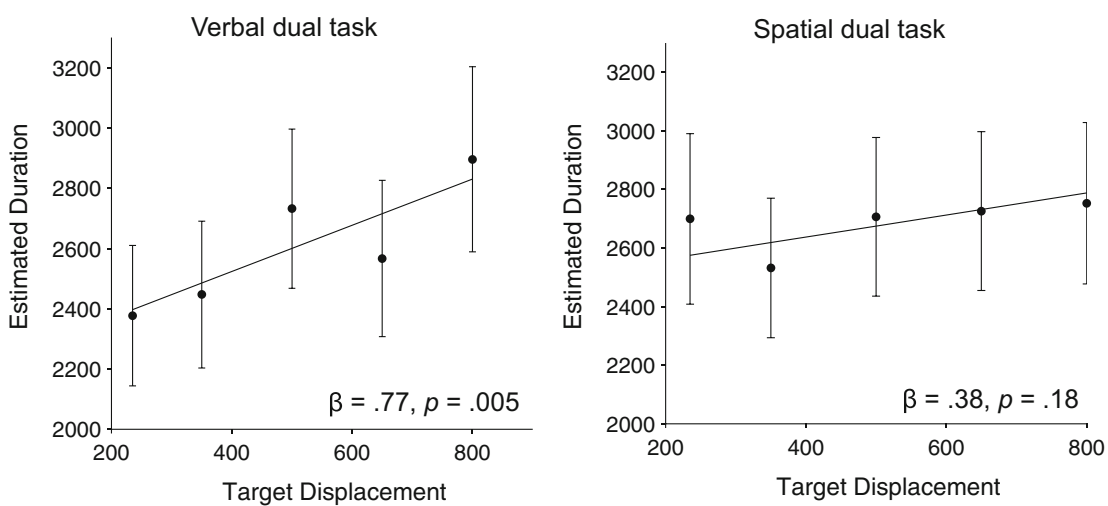
Table 1 Mean regression line slopes for each of the conditions. Parentheses contain standard deviations. Asterisks indicate that the slope is significantly greater than zero

\begin{tabular}{llll}
\hline & Standard condition & Verbal dual task & Spatial dual task \\
\hline Length & $1.03 *(.34)$ & $0.78 *(.35)$ & $.21(.28)$ \\
Duration & $0.013(.009)$ & $0.012(.008)$ & $.005(.01)$ \\
\hline
\end{tabular}

information. To probe the type of representations activated during this task, and therefore the underlying cause of this asymmetry, we had participants perform the reproduction task while performing verbal and spatial dual tasks to determine the effect of increasing verbal versus visuospatial working memory load.

We hypothesized that if the asymmetry in spatiotemporal interference stems from online linguistic processes, then a verbal dual task would reduce the influence of space on time whereas a spatial dual task would have no effect. Note that because line duration and spatial length are varied orthogonally in the present study and in Casasanto and Boroditsky (2008), the use of a covert verbal labeling technique (e.g., labeling the cued-dimension stimuli as "long" or "short") would result in no cross-dimensional interference. However, the verbal dual task enabled us to rule out the possibility that the asymmetric interference pattern is mediated by less explicit linguistic processes. Alternatively, if interference stems from conflicts related to visuospatial processing, then increasing visuospatial working memory load should minimize interference from irrelevant spatial information on temporal judgments. In support of this second hypothesis, we found that the asymmetric interference was preserved in the verbal dual task condition (see for Dolscheid, Shayan, Majid, \& Casasanto, 2013 for a similar finding regarding space-pitch mappings) but attenuated in the spatial dual task condition. This suggests that at least with respect to line reproduction tasks, the relative level of influence of spatial length on estimated duration is influenced by the capacity of visuospatial working memory.

One useful lens for interpreting these results is the load theory of attention (Lavie, Hirst, de Fockert, \& Viding, 2004). According to load theory, when cognitive load is relatively low, extraneous perceptual information is processed automatically, even when it is task irrelevant, and can therefore interfere with task-relevant information processing. With respect to the present results, it appears than in the standard and verbal dual task conditions, spatial information is processed even when it is irrelevant, rendering it available to cause it to interference with temporal judgments. However, in the spatial dual task condition, the load on visuospatial working memory may be sufficiently high to exhaust the capacity to process additional irrelevant perceptual information. In this condition, because spatial information is not processed when it is taskirrelevant, it cannot interfere with duration representations.
Of note is that although the spatial dual task did eliminate interference from line length on estimated duration, there was never an interference effect of line duration on estimated length. This suggests that even when the load on visuospatial working memory is low, irrelevant temporal information is not processed automatically. This is consistent with the many previous studies that have failed to find interference from temporal information on spatial judgments (Bottini \& Casasanto, 2010, 2013; Casasanto et al., 2010; Casasanto \& Boroditsky, 2008; V. Dormal \& Pesenti, 2012). Acuity for visually presented spatial extent is greater than acuity for temporal durations (Droit-Volet, Clément, \& Fayol, 2008), and it is been proposed that spatial information is processed more automatically than temporal information (V. Dormal \& Pesenti, 2012). This could explain why irrelevant spatial information is more likely to be processed than irrelevant temporal information. However, relative differences in processing automaticity may vary by modality. A recent study investigating temporal and spatial representations in the haptic dimension also reported an asymmetric pattern of interference, but in the opposite direction (Cai \& Connell, 2015). In this study, participants held sticks of varying lengths between their index fingers for various temporal durations, and the durations were accompanied by an auditory tone. When participants could only touch the sticks but not view them, judgments of the sticks' lengths were influenced by temporal duration, but judgments of the duration for which they held the sticks was not influenced by the length of the sticks. In this paradigm, temporal information (which is presented both haptically and aurally) may be more salient than the haptic spatial information, making it more likely to be processed when task-irrelevant and therefore able to cause interference. Therefore, it remains an open question as to whether the asymmetry present in processing visual spatial and temporal information persists when the information is presented in other modalities.

We believe that differences in processing automaticity are the most parsimonious explanation of asymmetric patterns of interference between dimensions, but this explanation is agnostic as to the source of these differences. It is likely that cultural factors, including language, may alter the relative saliency of spatial and temporal information. Notably, the asymmetry is not present in non-human primates: in monkeys, both spatial and temporal judgments are biased by irrelevant information from the other dimension (Merritt, Casasanto, \& Brannon, 2010). Furthermore, the specific form of spatiotemporal metaphors used by a language can alter which types of spatial information interfere with temporal reasoning (Boroditsky, 2001; Bottini \& Casasanto, 2013; Casasanto et al., 2010; Casasanto \& Boroditsky, 2008), which suggests that metaphors may mark particular dimensions as being especially salient. Additional work is therefore needed to determine how language and other factors affect the automaticity with which different magnitudes are processed. To this end, 
developmental work should investigate the emergence of asymmetric interference patterns between infancy and early childhood.

\section{Conclusions}

Representations of time are often found to be asymmetrically dependent on representations of space. In the present study, we confirmed that this asymmetry does not require online access to linguistic processing, as the pattern of asymmetric interference in a line reproduction task was preserved with a verbal dual task. However, when participants performed a spatial dual task, the asymmetry vanished, and the effect of irrelevant spatial information on temporal estimates was greatly attenuated. This suggests that the asymmetric pattern of interference observed between representations of space and time stems from differences in the automaticity with which spatial and temporal information are processed. As a result, asymmetric interference between spatial and temporal information may occur only when the cognitive demands on visuospatial working memory are low.

Acknowledgments This research was supported by an NSF Graduate Research Fellowship to AS and a James S. McDonnell Foundation Scholar Award and NSF Grant 0951690 from Research and Evaluation on Education in Science and Engineering and Developmental and Learning Sciences to EMB.

\section{References}

Allman, M. J., Pelphrey, K. A., \& Meck, W. H. (2012). Developmental neuroscience of time and number: Implications for autism and other neurodevelopmental disabilities. Frontiers in Integrative Neuroscience. doi:10.3389/fnint.2012.00007/abstract

Boroditsky, L. (2000). Metaphoric structuring: Understanding time through spatial metaphors. Cognition, 75(1), 1-28.

Boroditsky, L. (2001). Does language shape thought?: Mandarin and english speakers' conceptions of time. Cognitive Psychology, 43(1), 1-22. doi:10.1006/cogp.2001.0748

Bottini, R., \& Casasanto, D. (2010). Implicit spatial length modulates time estimates, but not vice versa. In S. Ohlsson \& R. Catrambone (Eds.), (pp. 1348-1353). Presented at the Proceedings of the 32nd Annual Conference of the Cognitive Science Society, Austin: Springer.

Bottini, R., \& Casasanto, D. (2013). Space and time in the child's mind: metaphoric or ATOMic? Frontiers in Psychology, 4. doi:10.3389/ fpsyg.2013.00803/abstract

Cai, Z. G., \& Connell, L. (2015). Space-time interdependence: Evidence against asymmetric mapping between time and space. Cognition, 136(C), 268-281. doi:10.1016/j.cognition.2014.11.039

Cantlon, J. F., Platt, M. L., \& Brannon, E. M. (2009). Beyond the number domain. Trends in Cognitive Sciences, 13(2), 83-91.

Casasanto, D. (2008). Who's afraid of the big bad Whorf? Crosslinguistic differences in temporal language and thought. Language Learning, 58(Suppl. 1), 63-79.
Casasanto, D., \& Boroditsky, L. (2008). Time in the mind: Using space to think about time. Cognition, 106(2), 579-593. doi:10.1016/j. cognition.2007.03.004

Casasanto, D., Fotakopoulou, O., \& Boroditsky, L. (2010). Space and time in the child's mind: Evidence for a cross-dimensional asymmetry. Cognitive Science, 34(3), 387-405.

Cohen Kadosh, R., Cohen Kadosh, K., Linden, D. E. J., Gevers, W., Berger, A., \& Henik, A. (2007). The brain locus of interaction between number and size: A combined functional magnetic resonance imaging and event-related potential study. Journal of Cognitive Neuroscience, 19(6), 957-970. doi:10.1162/jocn.2007.19.6.957

Cohen Kadosh, R., Henik, A., Rubinsten, O., Mohr, H., Dori, H., van de Ven, V., ... Linden, D. E. J. (2005). Are numbers special?: The comparison systems of the human brain investigated by fMRI. Neuropsychologia, 43(9), 1238-1248. doi:10.1016/j. neuropsychologia.2004.12.017

Cohen Kadosh, R., Lammertyn, J., \& Izard, V. (2008). Are numbers special? An overview of chronometric, neuroimaging, developmental and comparative studies of magnitude representation. Progress in Neurobiology, 84(2), 132-147.

Dolscheid, S., Shayan, S., Majid, A., \& Casasanto, D. (2013). The thickness of musical pitch: Psychophysical evidence for linguistic relativity. Psychological Science, 24(5), 613-621. doi:10.1177/ 0956797612457374

Dormal, V., Dormal, G., Joassin, F., \& Pesenti, M. (2011). A common right fronto-parietal network for numerosity and duration processing: An fMRI study. Human Brain Mapping, 33(6), 1490-1501. doi: $10.1002 / \mathrm{hbm} .21300$

Dormal, V., \& Pesenti, M. (2012). Processing numerosity, length and duration in a three-dimensional Stroop-like task: Towards a gradient of processing automaticity? Psychological Research. doi:10.1007/ s00426-012-0414-3

Droit-Volet, S., Clément, A., \& Fayol, M. (2008). Time, number and length: Similarities and differences in discrimination in adults and children. The Quarterly Journal of Experimental Psychology, 61(12), 1827-1846. doi:10.1080/17470210701743643

Feigenson, L. (2007). The equality of quantity. Trends in Cognitive Sciences, 11(5), 185-187.

Fias, W., Lammertyn, J., Reynvoet, B., Dupont, P., \& Orban, G. A. (2003). Parietal representation of symbolic and nonsymbolic magnitude. Journal of Cognitive Neuroscience, 15(1), 47-56. doi:10. 1006/nimg.2000.0697

Hubbard, E. M., Piazza, M., Pinel, P., \& Dehaene, S. (2005). Interactions between number and space in parietal cortex. Nature Reviews Neuroscience, 6(6), 435-448. doi:10.1038/nrn1684

Lakoff, G., \& Johnson, M. (1980). Metaphors we live by. Chicago: The University of Chicago Press.

Lavie, N., Hirst, A., de Fockert, J. W., \& Viding, E. (2004). Load theory of selective attention and cognitive control. Journal of Experimental Psychology: General, 133(3), 339-354.

Merritt, D. J., Casasanto, D., \& Brannon, E. M. (2010). Do monkeys think in metaphors? Representations of space and time in monkeys and humans. Cognition, 117(2), 191-202. doi:10.1037/0096-3445. 133.3.339

Santiago, J., Roman, A., \& Ouellet, M. (2011). Flexible foundations of abstract thought: A review and a theory. In A. Maas \& T. Schubert (Eds.), Spatial dimensions of social thought (pp. 39-108). Berlin: Mouton de Gruyter.

Srinivasan, M., \& Carey, S. (2010). The long and the short of it: On the nature and origin of functional overlap between representations of space and time. Cognition, 116(2), 217-241.

Tzelgov, J., Meyer, J., \& Henik, A. (1992). Automatic and intentional processing of numerical information. Journal of Experimental Psychology: Learning, Memory, and Cognition, 18(1), 166-179. doi:10.1037/0278-7393.18.1.166 
Walsh, V. (2003). A theory of magnitude: Common cortical metrics of time, space and quantity. Trends in Cognitive Sciences, 7(11), 483-488.

Winawer, J., Witthoft, N., Frank, M. C., Wu, L., Wade, A. R., \& Boroditsky, L. (2007). Russian blues reveal effects of language on color discrimination. Proceedings of the National Academy of Sciences, 104(19), 7780-7785. doi:10.1073/pnas.0701644104

Xuan, B., Zhang, D., He, S., \& Chen, X. (2007). Larger stimuli are judged to last longer. Journal of Vision, 7(10), 2. 